\title{
Sarcoidosis subcutánea en paciente con infección crónica por el virus de la hepatitis $C$
}

\begin{abstract}
Marina Fayos ${ }^{1}$, José M. Olmos-Martínez², Remigio Mazorra ${ }^{3}$, Jose D. García-Palacios'1, José M. Olmos
${ }^{1}$ Servicio de Medicina Interna. Hospital Universitario Marqués de Valdecilla-IDIVAL. Universidad de Cantabria. Santander (Cantabria). España

${ }^{2}$ Servicio de Aparato Digestivo. Hospital Universitario Marqués de Valdecilla-IDIVAL. Universidad de Cantabria. Santander (Cantabria). España ${ }^{3}$ Servicio de Anatomía Patológica. Hospital Universitario Marqués de Valdecilla-IDIVAL. Universidad de Cantabria. Santander (Cantabria). España
\end{abstract}

Recibido: 16/06/2018

Aceptado: 04/10/2018

En línea: 31/12/2018

Citar como: Fayos M, Olmos-Martínez JM, Mazorra R, García-Palacios JD, Olmos JM. Sarcoidosis subcutánea en paciente con infección crónica por el virus de la hepatitis C. Rev Esp Casos Clin Med Intern (RECCMI). 2018 (Dic); 3(3): 110-112. doi: 10.32818/reccmi.a3n3a3.

Autor para correspondencia: José M. Olmos. miromj@humv.es

\begin{tabular}{l} 
Palabras clave \\
\hline$\triangleright$ Sarcoidosis subcutánea \\
$\triangleright$ VHC \\
$\triangleright$ Interferón \\
$\triangleright$ Ribavirina \\
$\triangleright$ Boceprevir
\end{tabular}

Keywords

$\triangleright$ Cutaneous sarcoidosis

$\triangleright$ Hepatitis C virus infection

$\triangleright$ Interferon

$\triangleright$ Ribavirin

$\triangleright$ Boceprevir

\begin{abstract}
Resumen
La sarcoidosis subcutánea o enfermedad de Darier-Roussy es una variante muy poco frecuente de esta enfermedad. No se conoce bien su etiología, aunque se han descrito algunos casos en pacientes con infección crónica por virus de la hepatitis $\mathrm{C}(\mathrm{VHC})$, especialmente tras recibir tratamiento con interferón. Presentamos el caso de una paciente con infección por VHC que desarrolló una sarcoidosis subcutánea pocos meses después de recibir tratamiento con interferón, rivabirina y boceprevir. A pesar de que podría tratarse de una asociación casual, convendría estudiar el posible papel del VHC y del tratamiento con interferón en la etiopatogenia de esta enfermedad.

Abstract
Subcutaneous sarcoidosis (Darier-Roussy disease) is a very rare variant of this disease. Sarcoidosis is a disease
which still has uncertain etiology, although some cases have been described in patients with chronic HCV infec-
tion, especially after receiving treatment with interferon. We present the case of a patient with HCV infection who
developed a subcutaneous sarcoidosis a few months after receiving treatment with Interferon, Rivabirine and Bo-
ceprevir. Although it could be a casual association, it would be advisable to study the possible role of HCV and
treatment with IFN in the pathogenesis of this disease.
\end{abstract}

Puntos destacados

$\triangleright$ La sarcoidosis subcutánea es una variante muy poco frecuente de esta enfermedad.

$\triangleright$ La asociación de sarcoidosis en pacientes con virus de la hepatitis C tratados con interferón plantea la posibilidad de que dicho virus y el tratamiento con interferón intervengan en la etiopatogenia de esta enfermedad.

\section{Introducción}

La sarcoidosis es una enfermedad granulomatosa sistémica que afecta preferentemente a los pulmones y ganglios linfáticos. Alrededor del $25 \%$ de los casos presentan lesiones cutáneas que pueden preceder a las manifestaciones sistémicas. Las lesiones más comunes son placas, pápulas, eritema nodoso (lesión más característica y sugerente de actividad aguda de la enfermedad)', lesiones asociadas a cicatrices, lupus pernio y nódulos subcutáneos o enfermedad de Darier-Roussy. Esta entidad se ha descrito en contadas ocasiones ${ }^{2}$ y se manifiesta como nódulos firmes, móviles, indoloros, revestidos de piel normal, apreciándose en el estudio histológico la existencia de paniculitis lobulillar con granulomas no necrotizantes en dermis profunda e hipodermis. Suelen localizarse en extremidades $y$, con menor frecuencia, en tronco y cara. Su resolución suele ser espontánea en meses o años, pero también responden favorablemente al cabo de pocos meses al tratamiento con corticoides, aunque éstos no sean capaces de modificar el curso de la enfermedad.

En los últimos años, se han descrito una serie de casos de sarcoidosis en pacientes con infección por el virus de la hepatitis $C(\mathrm{VHC})$, que se desarrollan preferentemente tras instaurar tratamiento con interferón (IFN) ${ }^{3}$.

Recientemente, hemos tenido la oportunidad de estudiar a una paciente con infección por VHC que desarrolló un cuadro compatible con una sarcoidosis subcutánea tras recibir tratamiento con IFN, rivabirina (RVB) y boceprevir. Por ello, nos ha parecido de interés su comunicación.

\section{Historia clínica, anamnesis y pruebas complementarias}

Mujer de 53 años, con antecedentes de hipertensión arterial y síndrome ansioso-depresivo que ingresa en Medicina Interna por dolor lumbar y lesiones 
Fayos M, Olmos-Martínez JM, Mazorra R, García-Palacios JD, Olmos JM. Sarcoidosis subcutánea en paciente con infección crónica por el virus de la hepatitis $C$

nodulares subcutáneas. No refería hábitos tóxicos ni conductas de riesgo. A los 37 años fue diagnosticada de infección por VHC. Inicialmente, rechazó el tratamiento, aunque 6 años después acudió de nuevo a consulta siendo diagnosticada de hepatopatía crónica por VHC, genotipo 1b. Se inició tratamiento con IFN pegilado y RVB (6 meses), alcanzando una respuesta viral sostenida. Un año después de finalizar el tratamiento, se apreció una recaída bioquímica y virológica, por lo que se propuso una nueva tanda terapéutica que la paciente rehusó, no volviendo a la consulta hasta 5 años después. Dos años antes de ingresar en Medicina Interna, se instauró tratamiento con triple terapia (IFN, RVB y boceprevir), que tuvo que suspenderse a las 26 semanas por neutropenia, obteniéndose de nuevo una respuesta viral sostenida. Dos meses antes de su ingreso, comienza con dolor lumbar derecho, irradiado a epigastrio y mesogastrio, continuo, asociado a náuseas y vómitos, por lo que acudió varias veces al Servicio de Urgencias. También refería astenia, anorexia y pérdida no cuantificada de peso, así como la aparición de varios nódulos en extremidades.

En la exploración, se palpaban varios nódulos de consistencia firme, dolorosos a la palpación, de 1-2 cm de diámetro, en flexuras de ambos codos, región glútea y zona dorsal de la mano derecha. El resto de la exploración era anodina. El hemograma completo, la VSG, la bioquímica rutinaria, incluida la calcemia, y el elemental y sedimento de orina fueron normales. Los niveles de la enzima conversora de la angiotensina (ECA) se encontraban en el límite alto de la normalidad (87,1 U/l; N: 20-95 U/I). La carga viral del VHC fue indetectable y el estudio inmunológico (factor reumatoide, anticuerpos antinucleares, antipéptido citrulinado y anticitoplasma del neutrófilo) fue negativo, así como la detección de IFN gamma frente a Mycobacterium tuberculosis (Quantiferon ${ }^{\circledast}$ ). Las radiografías de tórax, abdomen, sacroilíacas y columna dorsal y lumbar no mostraron hallazgos de interés. El estudio ultrasonográfico mostró un hígado de tamaño normal, con bordes lisos y ecoestructura muy heterogénea, sin lesiones ocupantes de espacio, observándose en el fibroscan un grado de fibrosis avanzado (10,7 Kpa; F3). En la tomografía axial computarizada (TAC) toracoabdominal, se observaron adenopatías hiliares derechas sin infiltración parenquimatosa (Figura 1). En la resonancia magnética (RM) del codo derecho, se observó una lesión mal delimitada que infiltraba los tejidos adyacentes y que estaba muy vascularizada (Figura 2). Se realizó una biopsia por escisión de partes blandas de uno de los nódulos del brazo, apreciándose la existencia de una reacción granulomatosa de tipo sarcoideo, siendo el análisis molecular de Mycobacterium tuberculosis negativo (Figura 3).

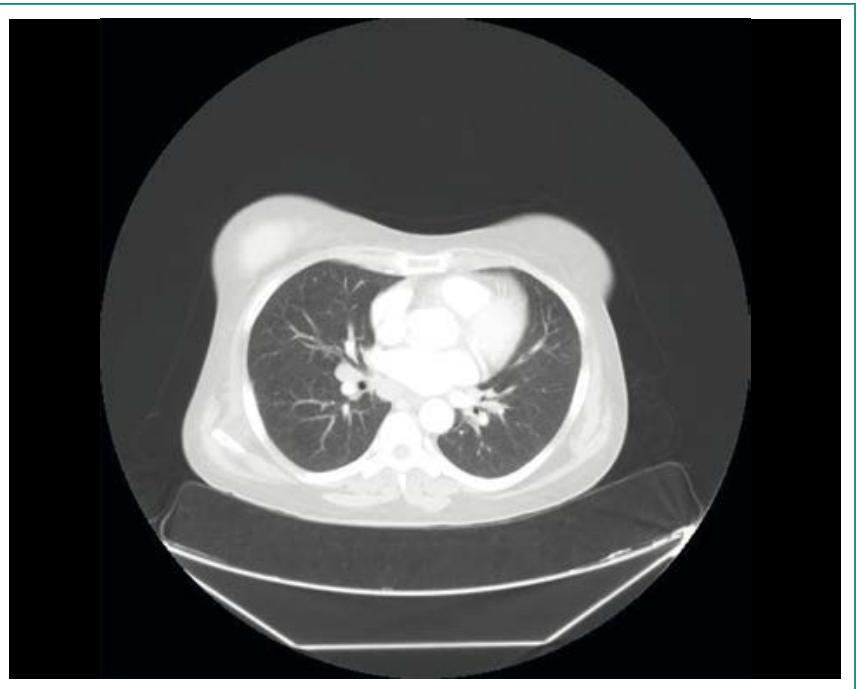

Figura 1. TAC torácica. Se observan adenopatías hiliares derechas sin infiltración parenquimatosa

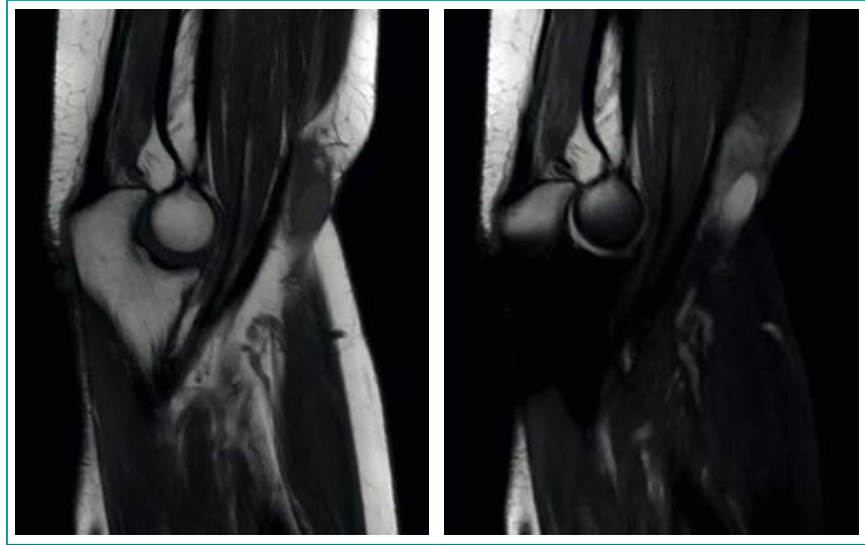

Figura 2. RM de codo derecho. Se aprecia la existencia de una lesión de $22 \times 17 \times 32$ mm, con bordes mal definidos, isointensa en T1 (izquierda) y moderadamente hiperintensa en $\mathrm{T} 2$ (derecha), que afecta a tejido celular subcutáneo e infiltra en profundidad el músculo braquial del antebrazo, que presenta realce homogéneo e intenso tras la administración de gadolinio

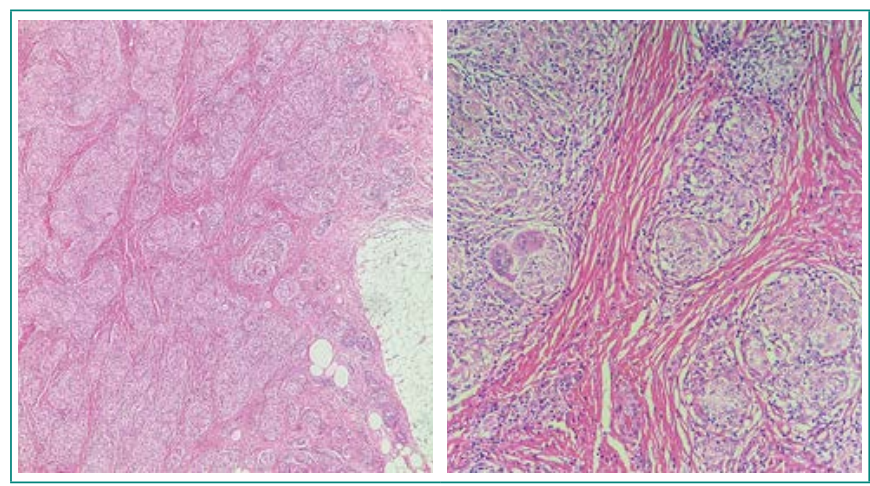

Figura 3. Biopsia cutánea (HE). Se observan la dermis y el tejido celular subcutáneo infiltrados por grupos de numerosos granulomas de pequeño tamaño (izquierda). A mayor aumento, se observan granulomas pequeños bien conformados, compuestos por células epitelioides y linfocitos pequeños, con células gigantes multinucleadas y presencia de cuerpos asteroides (granulomas de tipo sarcoideo) (derecha)

\section{Evolución}

La paciente recibió tratamiento con analgésicos y prednisona $(30 \mathrm{mg} / \mathrm{día}$ en pauta descendente). La evolución fue favorable, cediendo el dolor lumbar y desapareciendo los nódulos subcutáneos a los 2 meses de iniciarse el tratamiento. Un año después, la enferma se encuentra estable, sin nódulos y no existen datos bioquímicos ni virológicos de recaída.

\section{Discusión y conclusiones}

La sarcoidosis es una enfermedad granulomatosa sistémica que afecta preferentemente a pulmones, ganglios linfáticos, ojos y piel. No se conoce aún la etiopatogenia de esta enfermedad, aunque se ha asociado a factores genéticos, infecciosos, inmunológicos y ambientales ${ }^{1,2}$. La estimulación antigénica mediada por algunas partículas orgánicas e inorgánicas o por determinados virus, como por el ejemplo el VHC, y bacterias podrían activar las células T, con la consiguiente producción de citocinas (IL 2, INF gamma y TNF alfa) que estimularían la respuesta Th1 y la formación de granulomas ${ }^{3,4}$. Esta respuesta se ve favorecida en individuos genéticamente predispuestos, 
Fayos M, Olmos-Martínez JM, Mazorra R, García-Palacios JD, Olmos JM.

como ocurre con los que expresan antígenos codificados por el HLA-DRB1 y HLA-DQ15,6.

El VHC se ha relacionado con numerosas manifestaciones extrahepáticas de base inmunoinflamatoria, siendo la crioglobulinemia asociada a VHC la más representativa. Estas enfermedades asociadas al VHC presentan frecuentemente manifestaciones cutáneas, como sucede en la sarcoidosis. Además, la infección crónica por el VHC se asocia clásicamente con otras entidades dermatológicas como la porfiria cutánea tarda o el liquen plano, y también son relativamente frecuentes los efectos adversos secundarios al tratamiento, como las reacciones locales en el punto de inyección o los cuadros de dermatitis asociada.

En 1993, Blum et al.7 describieron el primer caso de asociación entre la infección por el VHC y la sarcoidosis, habiéndose descrito desde entonces alrededor de un centenar de casos en la literatura. En la mitad de ellos, aparecen manifestaciones cutáneas de la enfermedad, con menor tendencia a la afectación sistémica. Cabe destacar que el 75\% de los pacientes desarrolla la sarcoidosis tras comenzar el tratamiento con IFN³. Por ello, algunos autores han sugerido que la infección por VHC provocaría una disregulación de la red de citocinas asociadas a la respuesta Th1 en pacientes predispuestos genéticamente que favorecería formación de granulomas ${ }^{4}$. En este contexto, la administración de IFN podría potenciar esta respuesta al favorecer la actividad Th1 y disminuir la Th25.

Tal y como sucedió en el caso que describimos, la sarcoidosis inducida por IFN puede presentarse hasta 30 meses después de iniciarse el tratamiento, en forma de enfermedad cutánea o pulmonar 6 . Predomina en mujeres con una edad media de 50 años, y clínicamente puede acompañarse de manifestaciones generales (astenia, anorexia, adelgazamiento) similares a las que aparecen en ocasiones tras la terapia con IFN', tal y como ocurrió en nuestra enferma. Habitualmente, las lesiones sólo aparecen si el IFN se combina con la RVB, ya que ésta también produce citocinas tipo 1, pero no se han descrito cuando se administra RVB de forma aislada. Nuestra paciente recibió inicialmente tratamiento con IFN y RVB, pero fue posteriormente, tras administrar la triple terapia (IFN, RVB y boceprevir) cuando desarrolló la afectación cutánea, lo que plantea la posibilidad de que la exposición reiterada al tratamiento combinado con IFN y RVB haya potenciado la disregulación inmune, favoreciendo la aparición de los granulomas cutáneos.

La sarcoidosis subcutánea inducida por estos medicamentos suele tener buen pronóstico, salvo cuando la enfermedad ya estaba presente antes de iniciarse el tratamiento. Habitualmente, se autolimita en pocos meses sin necesidad de suspender el tratamiento con IFN, aunque puede ser necesario asociar esteroides locales o sistémicos ${ }^{7}$. En estos casos, debe monitorizarse la función hepática, la carga viral de VHC y el recuento celular³. Además de los corticoides, se han utilizado también otros fármacos, como hidroxicloroquina, colchicina, metrotrexato, anti-TNF-alfa, minociclina, talidomida e isotretinoína ${ }^{3,8}$.

A pesar de los casos de sarcoidosis en pacientes con infección por VHC descritos en la literatura, no se ha analizado aún la prevalencia de la infección por el VHC en pacientes con sarcoidosis. Aunque podría tratarse de una asociación casual, convendría estudiar el posible papel del VHC y del tratamiento con IFN en la etiopatogenia de esta enfermedad, si bien cabe esperar que con las nuevas terapias antivirales de acción directa, esta manifestación extrahepática sea cada vez menos frecuente al caer el IFN en desuso.

\section{Bibliografía}

1. Ungprasert P, Wetter DA, Crowson CS, Matteson EL. Epidemiology of cutaneous sarcoidosis, 1976-2013: a population-based study from Olmsted County, Minnesota. J Eur Acad Dermatol Venereol. 2016 Oct; 30(10): 17991804. doi: 10.1111/jdv.13760.

2. Ando M, Miyazaki E, Hatano $Y$, Nishio S, Torigoe C, Yamasue M, et al. Subcutaneous sarcoidosis: a clinical analysis of nine patients. Clin Rheumatol. 2016 Sep; 35(9): 2277-2281. doi: 10.1007/s10067-016-3356-0.

3. Ramos-Casals M, Mañá J, Nardi N, Brito-Zeron P, Xaubet A, Sánchez-Tapias $J M$, et al. Sarcoidosis in patients with chronic hepatitis $C$ virus infection: analysis of 68 cases. Medicine (Baltimore). 2005; 84: 69-80.

4. Bonnet F, Morlat P, Dubuc J, De Witt S, Bonarek M, Bernard N, et al. Sarcoidosis-associated hepatitis C virus infection. Dig Dis Sci. 2002; 47: 794-796.

5. Fallahi P, Ferri C, Ferrari SM, Corrado A, Sansonno D, Antonelli A. Cytokines and HCV-related disorders. Clin Dev Immunol. 2012; 2012: 468107. doi 10.1155/2012/468107.

6. Rogers CJ, Romagosa R, Vincek V. Cutaneous sarcoidosis associated with pegylated interferon alfa and ribavirin therapy in a patient with chronic hepatitis C. J Am Acad Dermatol. 2004; 50: 649-650.

7. Blum L, Serfaty L, Wattiaux MJ, Picard O, Cabane J, Imbert JC. Nodules hypodermiques sarcoidosiques au cours d'une hepatite virale $C$ traitee par interferon alpha 2b. Rev Med Interne. 1993; 14(Suppl 462): 1161.

8. Ramírez C, Faúndez C, Valdés MP, de la Fuente R, Carreño L. Sarcoidosis subcutánea como manifestación inicial de sarcoidosis sistémica. Piel (Barc). 2013; 28: 387-390. doi.org/10.1016/j.piel.2013.01.015. 